\title{
EVOLUTION OF THE Design ENGINEERING MENTORSHIP PROGRAM
}

\author{
Kush Bubbar, Alexandros Dimopolous, Roslyn Gaetz, Peter Wild and Michael McWilliam \\ Faculty of Engineering, University of Victoria, BC, Canada \\ Corresponding Author: kbubbar@uvic.ca
}

\begin{abstract}
The Design Engineering Mentorship Program (DEMP) is a five-day intensive training program focused on developing appropriate competencies in graduate students required to effectively teach engineering design at the undergraduate level.

Evolution of the present program is discussed in context of feedback and observations from the now defunct Design Engineering \& Instruction program. The structure of the procedural based DEMP program is fully described including new experiential based workshops on creativity and coaching led by a PCC certified coach.

Motivating factors and implementation details of each of the workshops are described in detail in context of the competencies attributed to a design instructor.

The first instance of the DEMP program will be offered in September 2016.
\end{abstract}

Keywords: engineering design, coaching, creativity through divergent inquiry, teaching assistants, workshops, graduate students

\section{INTRODUCTION}

The research in educational psychology states that the competencies required to perform engineering design reside in the upper levels of Bloom's taxonomy in the cognitive domain (i.e. Analysis, Evaluation, and Synthesis). In contrast the majority of engineering instruction in academia takes place at the lower levels (Knowledge, Comprehension, and Application) leading to a competency gap in engineering graduates [1].

Teaching engineering design in undergraduate curricula is a systemic issue at most academic institutions. The issue stems from the complexity of teaching the associated higher level competencies and their subsequent assessment which often leads to substantial resources for successful implementation [2].

The University of Victoria was awarded one of the NSERC Chairs in Design Engineering (CDE) with a mandate to improve engineering design instruction. Both the Design Engineering \& Instruction (DE\&I) as well as the successor Design Engineering Mentorship Program (DEMP) are initiatives born out of this mandate.

The mandate of the DEMP program is to develop these higher level cognitive competencies in graduate students, which are critical to fostering their capabilities as future Design Teaching Assistants (DTAs), through execution of an experiential learning program where they develop and deliver a design project to a group of undergraduate students.

This article will discuss the history behind the DE\&I program and the motivating factors for its evolution into the DEMP program, followed by a detailed description of DEMP program structure. The rationale behind each of the individual workshops will be examined along with the final design project challenge. The first delivery of the DEMP program will be in September 2016.

\section{PROGRAM HISTORY}

Under the mandate of the NSERC Chair in Design Engineering (CDE), the University of Victoria sought to increase undergraduate students' exposure to engineering design by augmenting conventional engineering science courses with mini design projects. This was to be in addition to the traditional cornerstone and capstone courses.

The primary issue with implementing the mini design projects was the significant time investment for course instructors. An innovative question was considered: Could graduate students be employed to generate and administer these mini design projects? This led to an ensuing challenge; the strength of graduate student design competencies varied greatly. The DE\&I program was created to address these deficiencies.

\subsection{Design Engineering \& Instruction}

The scope of the DE\&I program was to develop and train Design Teaching Assistants (DTAs) on the following basic competencies of design engineering [3]: 
1. Stages of the Design Process

2. Open Minded

3. Communication

4. Project Coaching

5. Teamwork

The training was delivered through a combination of workshops, discussion panels, seminars, and a capstone project in which the DTAs would develop and deliver small design challenge to a group of high school students.

The competencies of the participants were to be assessed upon completion of the training. Those with the appropriate combination of domain knowledge and a minimum threshold design competency could then be identified and tasked with developing appropriate mini design projects.

The results of the DE\&I program were met with moderate success. Most of the DTAs exhibited less design experience than was originally expected. Some had no formal design training whatsoever [3]. This was a serious issue since teaching assistants unskilled in the basic engineering design competencies would be unable to create and administer the envisioned design projects.

The DEMP program was created to address the shortcomings of the DE\&I program with two additional primary drivers:

- $\quad$ Identifying graduate students with existing strong engineering design competencies for future recommendation as teaching assistants in design curriculum.

- Offering an experiential training program to introduce new graduate students to engineering design and "plant a seed”.

\subsection{Design Engineering Mentorship Program}

The evolution of the DE\&I program into the DEMP program was the result of the motivations described above alongside feedback from both the DTAs as well as observations from the program instructors. The feedback and observations are summarized below:

2.2.1 Positive Feedback and Observations Positive feedback and observations from the DE\&I program are listed below:

1. Developing and delivering the design project challenge was extremely effective at teaching the DTAs about the design process.

2. Pre-workshop readings, summarizing activity, and formative feedback prior to program commencement was extremely helpful in minimizing misconceptions.

3. High school students enjoyed the hands-on experience of working through several design projects.

2.2.2 Opportunities for Improvement Listed below are opportunities for improving the DE\&I program:

1. DTAs struggled with coaching / mentoring the high school students under "unexpected" circumstances (e.g. dominant student in a group).

2. DTAs struggled differentiating between design and science projects.

3. Not enough time was allocated for exploring ideas for design project challenges.

4. No framework was offered for developing the ideas

5. DTAs often hit blockages during the development of their design project challenge with little knowledge on how to proceed.

6. DTAs relied heavily on the internet as a source of ideas. This was a crutch for ideation.

7. Seminars from external professionals were disjointed from the learning outcomes of the program.

It was clear DTAs lacked the basic knowledge and skills to coach students through issues with team dynamics as well as supporting student-led decision making. In addition, the DTAs themselves may have understood the basics about the design process but had little ability to traverse the process. Finally, DTAs did not have much practice with creative thinking processes resulting in an overreliance on the internet for project ideas.

On the other hand, both the DTAs and high school students thoroughly enjoyed the experience of delivering and executing the design projects, leading to retention of this aspect in the DEMP program. These considerations were taken into account during the redesign of our program.

\section{DEMP PROGRAM STRUCTURE}

Through reflection of the shortcomings of the DE\&I program, a picture of a Design Instructor emerged as an individual with: Knowledge of the Design Process, Ability to Coach students and teams to deter poor performance, Ability to spark Creative thought, and possess strong Domain Knowledge on the problem at hand as detailed in Fig. 1. 


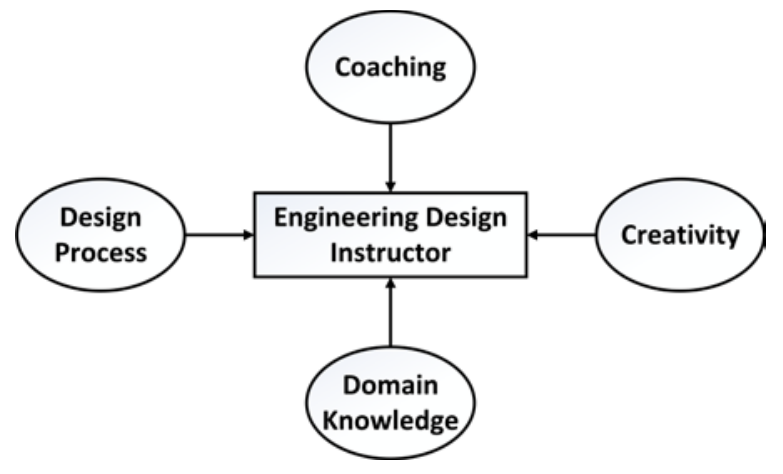

Fig. 1. Competencies of an Engineering Design Instructor.

The Design Engineering Mentorship Program was established as a five-day training program operating six hours per day as a basis to mold this persona. Upon completion of the program DTAs should possess components of the first three competencies while the forth (Domain Knowledge) is the unique attribute each graduate students brings to the table.

A week prior to the workshop a short Reading Assignment will be delivered for DTAs to familiarize themselves with the basics of engineering design.

The first day and a half of the program is dedicated to delivering experiential based workshops which address the first three roles of the Engineering Design Instructor:

1. Basics of Engineering Design Process

2. Creativity through Divergent Inquiry

3. Coaching for Success

The following two and a half days then focus on the systematic development, execution, iterative testing and reflection of the DTAs design project challenge and their project pitch employing the skills introduced in the workshops.

The design project challenge is then delivered on the morning of the final day to a group of undergraduate students. Feedback on the program, instructors and DTAs along with a guided reflection period is scheduled in the afternoon. Each component of the program is detailed in the following subsections.

\subsection{Reading Assignment and Formative Feedback}

A reading assignment will be sent to participants one week before the start of the program. It will introduce Hyman's design process [4] and emphasize:

- Differences in the attributes of engineering science versus engineering design problems
- Importance of the engineering design process

- Systematic formulation of the Need, Problem Statements, Objectives, and Constraints

DTAs are asked to:

- Describe the important features of both engineering science and engineering design problems

- Detail Hyman's nine stage design process describing the features and goals of each stage

- Define the Need and all the elements of the Problem Statement

- Practice generating Problem Statements from contextualized situations

The reading assignment is submitted prior to the first day of the program. Responses are reviewed by the instructors and formative feedback is delivered back to the DTAs so that conceptual misunderstandings can be caught before the program starts.

\subsection{Engineering Design Fundamentals}

In this first workshop, DTAs get to know one another and are introduced to the program and its goals. A group review of the distinctions between engineering science and engineering design ensues to reconnect with the material from the reading assignment.

To promote the distinction between engineering science and design problems, a group exercise is given to convert a science statement into an open-ended design problem [1]. For example, the science statement "measure the transmissivity coefficient of this window" becomes "how can the transmissivity coefficient be improved in this window without changing the footprint?"

Finally, the workshop concludes with the DTAs participating in a short design project administered by the instructors with a catch. The first instructor administers the project poorly by providing insufficient information surrounding the project requirements ending abruptly with no prompting. The second instructor then administers the same project with well-defined requirements. A reflective period ensues with the DTAs recognizing the importance of establishing a clear problem statement.

\subsection{Creativity through Divergent Inquiry}

Much research has been conducted highlighting the importance of creativity in engineering design [5]. This research indicates that creativity is not an intrinsic ability we are born with, but rather a skill which can be taught. 
The most effective methods to teach creativity are those which emphasize teaching cognitive frameworks [6].

Experts conceptualize creativity as a dynamic process employing the cognitive skill of divergent inquiry [7]. In Dym's famous publication he describes divergent inquiry as "attempts to diverge from facts to possibilities that can be created from them" which opposes the standard emphasis of engineering education. Dym further explains that "good designers" not only know how to apply divergent inquiry, but also know when to apply convergent inquiry [2].

Most graduate students possess strong convergent reasoning skills, hence this workshop will not emphasize teaching convergent thinking but rather prompt its usage at appropriate points. The workshop will teach divergent reasoning through completion of experiential based activities.

The goals of the creativity workshop are to stress the important idea that creativity can be taught, and to generate design project ideas through the application of a creative cognitive framework. Divergent reasoning is facilitated through execution of a guided brainstorming affinity technique in which the primary constraint is time to demote fixation and focus on the quantity of ideas generated.

The output of this workshop is the generation and selection of project themes by the DTAs guiding the development of the eventual design projects they will teach at the end of the week. Projects are created in a social environment with participants first working as individually to come up with three physical principles (e.g. heat transfer) in a space of three minutes. Each principle is written onto a blue cue card. All of the cards are then collated, refined, and randomly placed onto ten numerated chart sheets. Criticisms and outside references, such as the internet, are not permitted since the goal is to practice ideation.

The second step is to generate ideas related to the principles (ex. thermocouple, fan, fins are related to heat transfer). DTAs are randomly grouped and assigned a chart. The groups are given five minutes to generate five ideas related to their principles. Each idea is written onto a white cue card and affixed to the chart sheet. Groups are shuffled and the process is repeated until all ten charts are populated.

The third step is to begin the creation of a design problem. Groups are shuffled again, given ten minutes, and asked to sketch a situation utilizing the principles and ideas on the populated chart, hence applying convergent reasoning. For example, measuring the temperature of a metal plate using a thermocouple which is in contact with a heat source while heat is dissipated through fins.

The sketches are then converted into a problem statement by applying Hyman's problem definition methodology. For example, design a system to maintain a temperature of $40{ }^{\circ} \mathrm{C}$ on a metal place while it is in contact with a resistive heat source. The designer has at their disposal one metre of aluminum foil and a small fan. At last individuals vote and projects are assigned for the design project challenge.

\subsection{Coaching for Success}

It is well understood that engineering design is best taught in a Project Based Learning (PBL) environment in which students traverse a design process in teams while engaging in real world engineering problems [2]. Success depends on team dynamics along with learning how to make "good choices" in an ambiguous design process.

This is where the role of a coach is pivotal in facilitating students through the challenges of a PBL environment allowing for the best probability of success [8]. As discussed above, coaching is an important competency our graduate student population lacks. Previous DTAs from the DE\&I program recognize this and have voiced their desire to develop coaching skills.

The coaching workshop is structured to 1) Introduce the basic theory behind team dynamics and coaching; 2) Teach simple strategies on how to coach teams effectively; 3) Practice coaching strategies through experiential activities. The coaching workshop is facilitated by a PCC licensed coach with fifteen years of professional experience.

3.4.1 Introduction to Group Dynamics and Coaching In his famous article, Tuckman introduced a model which described group dynamics in terms of necessary phases (Forming, Storming, Norming, and Performing) a team needs to traverse to operate optimally [9]. The role of a coach is to support positive movement through the stages of group dynamics in order to promote mutual understanding, appreciation for diverse perspectives and competing frames of reference, and integration of other perspectives [10]. Using coaching strategies in teams, such as creating stem sentences and using coaching language, helps create and promote team innovation, commitment to team goals, and improved team results [11]. In particular, the "Storming" stage (or the "Groan Zone") is where personalities clash resulting in conflict. This conflict must be resolved before the team may operate effectively.

At the heart of the conflict is the mindset of the team. The Judger - Learner model is a Choice Model that describes two basic mindsets from which we can operate. 
It frames our view of the world and shapes our beliefs about limitations and possibilities. This practical tool can help individuals make more effective choices and increase collaboration [12]. Individuals using the Judger's mindset are usually reactive and automatic in their responses while those who access the Learner's mindset are solution seeking and try to create win/win opportunities and situations [13]. The role of the coach is to help facilitate the group dynamic in which team members and coach create a positive team environment that fosters curiosity, innovation, creativity, "buy in", and commitment from the team to see the project through [11].

Team performance is also based on making "good decisions.” Making good decisions involves domain knowledge and experience which many students do not possess. The role of the coach is to facilitate self-directed learning as opposed to the standard academic method of dispelling factual truths as in teacher-directed learning [8]. The coach helps students make those decisions but never forces a decision onto them.

3.4.2 Teaching Coaching Strategies The primary methodology taught in this workshop is the use of stem sentences. A stem sentence is an open-ended question asked by the coach to engage self-directed thought from the student. The use of stem sentences varies depending on context. An example used to broaden participation in a group setting is "who else feels similarly?" This stem sentence helps to identify team members with a similar perspective on a thought.

To begin with, DTAs will become familiar with a standard list of stem sentences and the typical contexts in which they are used. This is followed by an activity in which DTAs create their own domain specific stem sentences on a worksheet. In this regard DTAs learn how to use this technique as well as begin to create a personalized inventory.

Guidance on best practices are also a standard means of supporting student led decision making in design courses. DTAs will be provided a short list of generic engineering best practices for which they may use to prompt student self-directed learning. For example, "test early and test often."

\subsubsection{Practicing Coaching Strategies through Participation in Experiential Activities}

Four experiential based activities will be performed throughout the workshop.

\section{Activity 01 - Contrasting Facilitation Styles:}

The workshop commences via an activity with a twist. DTAs are divided into two groups with each group given a design project challenge. The instructors use a "good cop, bad cop" model in which one instructor uses open-ended questions and an inquiry based approach to supporting team members in their design. The other takes a directive and authoritative approach to support their team members. After ten minutes, the DTAs switch projects. Upon completion of both design challenges, DTAs debrief on their experiences. An example-rule methodology is used to expose DTAs to being coached prior to discussing the associated theory as a basis to invoke discovery based learning [14].

\section{Activity 02 - Puzzle Game:}

DTAs are divided into groups of four with one team member wearing a blindfold. The goal is for the blindfolded person to put together a simple puzzle while being coached by the other three team members. To succeed, team members will realize that they must communicate in a clear and supportive manner.

\section{Activity 03 - Creative Coaching Interactive Demo}

Through role-play both instructors attempt to complete a design challenge as a team. Difficulties arise during design challenges due to group interactions, differences in personality, the commitment to the project idea, and understanding of the problem. The coaching facilitator interacts with the DTAs to solicit suggestions on identifying the source of the conflict, seeks suggestions on how to increase collaboration and effective listening between team members, while building commitment towards an attainable goal. Creating an experiential roleplay simulation and providing the opportunity for reflective practice enhances the DTAs’ learning [15].

\section{Activity 04 - Creative Coaching Practice:}

Finally, the last activity involves the DTAs breaking into four groups with each provided a different design challenge. One person is assigned as the coach while the other three are the students. The coach is also supplied with a pre-defined solution to the design challenge. The goal for the activity is for the coach to effectively support students in developing a solution to the design challenge without pushing the suggested solution. This type of facilitation aligns with the self-directed learning model [16]. After ten minutes the teams rotate design challenges with another group member acting as the coach.

Through participation in the coaching workshop, DTAs will develop and practice using a repertoire of coaching strategies they can employ when they deliver their design project challenge.

\subsection{Development of the Design Project Challenge}

Development of the Design project challenge occurs over three consecutive days. 
3.5.1 Day One. Day one of the Design Project Challenge begins with the problem statement developed as the output of the creativity workshop. The problem statement is first systematically refined applying Hyman's method. Next each group is tasked with generating three feasible concepts in sketched form satisfying the original problem statement. A reflection period then commences to review and iterate both the concepts as well as the problem statement. The day concludes with the creation of a Bill of Materials (BOM).

3.5.2 Day Two. The goal of day 02 is for DTAs to build, assess, and iterate their design project challenge followed by delivering the project to another external group.

In the morning groups are tasked to build all three of their concepts using the purchased materials from their $\mathrm{BOM}$, assess the concepts against the design objectives and constraints, and finally iterate both the problem statement and concept designs. In the afternoon, groups generate a five-minute pitch to introduce their design challenge and finally take turns delivering their projects to one another. Upon completion of the external trials, feedback is provided by the members executing the design challenge on the: 1) clarity of the pitch, 2) execution of the design project (fun, challenging, etc...) and finally 3) quality of the coaching. This feedback is used to iterate the project and update the BOM.

3.5.3 Day Three. The goal of day 03 is for DTAs to refine their project. Teams are tasked with first rebuilding their concepts with the new BOM materials, then reflecting and iterating the problem statement. External trials once again proceed along with a reflection period for administering feedback. The final half day is dedicated as an open development session.

\subsection{Design Challenge Delivery and Reflection}

Projects are delivered to undergraduate teams of 4-5 students per team with 45 minutes allocated to executing the design project challenge and 15 minutes for reflection and feedback. Student teams are cycled until each project is executed three times.

The afternoon is dedicated as a period of reflection and feedback. DTAs complete online surveys assessing the performance of the DEMP program, instructors, their group as a whole, as well as themselves.

\section{CONCLUSIONS}

The innovative Design Engineering Mentorship Program was founded out of the necessity to foster competencies in graduate students appropriate for supporting undergraduate curriculum. Through detailed review of the feedback and observation of the DE\&I program, a specialized, process focused yet experiential based program was created. The first offering of the program is set to run in September 2016.

\section{Acknowledgements}

The authors would like to graciously acknowledge the Resource Centre for Students with a Disability at the University of Victoria for their time, commitment, and teaching and coaching expertise in supporting the development of this innovative program. We also appreciate the Natural Sciences and Engineering Research Council (NSERC) of Canada for their funding to administer the Design Engineering Mentorship Program.

\section{References}

[1] R. E. Terry and J. N. Harb, "Kolb, Bloom, creativity, and engineering design,” in ASEE Annual Conference Proceedings, 1993, vol. 2, pp. 1594-1600.

[2] C. L. Dym, A. M. Agogino, O. Eris, D. D. Frey, and L. J. Leifer, "Engineering Design Thinking, Teaching, and Learning,” J. Eng. Educ., vol. 94, no. 1, pp. 103-120, Jan. 2005.

[3] F. Firmani, M. McWilliam, P. Wild, M. McGuire, N. Dechev, and C. Bradley, "TRAINING PROGRAM IN ENGINEERING DESIGN FOR GRADUATE TEACHING ASSITANTS,” in Proceedings of the Canadian Engineering Education Association, 2013.

[4] B. I. Hyman, Fundamentals of engineering design. Prentice Hall, 2002.

[5] S. R. Daly, E. A. Mosyjowski, and C. M. Seifert, "Teaching Creativity in Engineering Courses,” J. Eng. Educ., vol. 103, no. 3, pp. 417-449, Jul. 2014.

[6] E. Karpova, S. B. Marcketti, and J. Barker, “The Efficacy of Teaching Creativity: Assessment of Student Creative Thinking Before and After Exercises,” Cloth. Text. Res. J., vol. 29, no. 1, pp. 52-66, Jan. 2011.

[7] Guilford, "Varieties of divergent production,” J. Creat. Behav., vol. 18, no. 1, pp. 1-10, 1984.

[8] J. W. Dally and G. M. Zhang, "A Freshman Engineering Design Course,” J. Eng. Educ., vol. 82, no. 2, pp. 83-91, Apr. 1993.

[9] B. W. Tuckman, "Developmental sequence in small groups," Psychol. Bull., vol. 63, no. 6, pp. 384-399, 1965.

[10] S. Kaner, Facilitator's guide to participatory decision-making. John Wiley \& Sons, 2014.

[11] V. Rousseau, C. Aubé, and S. Tremblay, "Team coaching and innovation in work teams: An examination of the motivational and behavioral intervening mechanisms," Leadersh. Organ. Dev. J., vol. 34, no. 4, pp. 344-364, 2013.

[12] Greenaway, D., Sinclair. M., Trowhill, A., \& Roy, L., “Coaching in the context of work," presented at the Adler Faculty of Professional Coaching Inc., Toronto, Ontario, 2013-2011.

[13] Goldberg, M. C ., “Choice Model: Learner vs Judger,” presented at the Coaching in the Context of Work. Adler Faculty of Professional Coaching Inc, Toronto, Ontario, 2002.

[14] J. T. Guthrie, "Expository instruction versus a discovery method.," J. Educ. Psychol., vol. 58, no. 1, p. 45, 1967.

[15] D. MacKeracher, Making sense of adult learning. University of Toronto Press, 2004.

[16] P. O. Ozuah, "First, there was pedagogy and then came andragogy,” 2005. 